\section{Poster abstracts}

\section{MEDICINE USE AND MEDICINE RELATED PROBLEMS IN PATIENTS WITH LIVER CIRRHOSIS: A SYSTEMATIC REVIEW OF QUANTITATIVE AND QUALITATIVE STUDIES}

${ }^{1}$ Ejaz Cheema, ${ }^{1}$ Abdullah Al-Hamid, ${ }^{2}$ Aliaa Al-Aryan. ${ }^{1}$ University of Birmingham; ${ }^{2}$ University of Hertfordshire

10.1136/postgradmedj-2018-fpm. 12

Introduction Liver cirrhosis is a major chronic disease that is associated with high morbidity and mortality. ${ }^{1}$ The associated co-morbidities and medical complications together with the use of complex and multiple drug therapies by patients with liver cirrhosis put them at an increased risk of developing medicine related problems (MRPs). MRPs can be defined as an event or circumstance involving drug therapy that actually or potentially interferes with desired health outcomes. ${ }^{2}$ This review aims to systematically investigate the prevalence, causes and risk factors of MRPs in cirrhotic patients and to explore factors influencing the medicine use from patients and health care providers' perspectives.

Subjects and methods Eight online databases (PubMed, Scopus, CINAHL, The British library, PsycINFO, Web of Science, EMBASE, and Google scholar) were searched up to 30th Sep, 2018 with no start date. Both quantitative studies including randomised controlled trials (RCTs), clinical trials and observational studies as well as qualitative studies were included in the review. Search terms used included 'medicine related problems', 'medicine use', 'patients' perceptions' and 'adverse drug reaction'. Data collected included the study design, baseline characteristics of study population and study outcomes. Appropriate Critical Appraisal Skills Programme (CASP) tools were used to assess both the quality of quantitative studies and qualitative studies. An in-depth descriptive analysis of the quantitative data and a thematic analysis of the qualitative data were undertaken.

Result 16 quantitative studies and 11 qualitative studies were included in the review. Of the 16 quantitative studies, nine examined the prevalence, causes, risk factors and medicine classes associated with MRPs and the remaining seven studies reported factors affecting medicine use. Mean frequency of MRPs reported in the quantitative studies ranged from 14\%23.4\%. The most frequent causes of MRPs identified in the studies included drug interactions, inappropriate dosing and use of contraindicated drugs. Major risk factors associated with MRPs included polypharmacy, severity of liver disease and length of hospital stay. Diuretics, analgesics and sedatives were suspected to be among the most common drug classes associated with MRPs. The thematic synthesis of qualitative data identified three key themes: patient related factors including lack of patients' knowledge about liver cirrhosis and its risk factors; health care related factors including lack of communication between healthcare professionals and patients; stigma and negativity including negative attitudes (misconceptions and discriminations) and negative consequences of liver cirrhosis.

Conclusions MRPs pose a significant social and financial burden on both patients and healthcare systems. Findings of this review suggest that the management of liver cirrhosis should not only be limited to the provision of safe and effective drug therapy but should also expand to improving the understanding and awareness of patients about the disease. Future research should focus on assessing the impact of patient- specific education on medicine use by patients with liver cirrhosis.

\section{REFERENCES}

1. Murray CJ, Vos T, Lozano R, et al. Disability-adjusted life years (DALYs) for 291 diseases and injuries in 21 regions, 1990-2010: a systematic analysis for the Global Burden of Disease Study 2010. Lancet 2012;380:2197-2223.

2. Strand L, Morley P, Cipolle R, Ramsey R, Lamsam G. Drug-related problems: their structure and function. DICP 1990;24:1093-1097.

\section{POMPHOLYX - A RARE SIDE EFFECT OF TRASTUZUMAB}

Abubakkar Raheel, Syed IIfan Wafa, Anupama Gore. University Hospitals of Leicester, Leicester Royal Infirmary

\subsection{6/postgradmedj-2018-fpm. 13}

Background Trastuzumab is a monoclonal antibody prescribed as part of systemic treatment of breast cancer. Trastuzumab can be prescribed on its own or as an adjuvant with chemotherapy or hormone therapy against over-expressing HER2 positive breast cancer. Patients on Trastuzumab can develop side effects of nausea, vomiting, cardiotoxicity and flu-like symptoms. ${ }^{1}$ However, we describe a rare side effect of Pompholyx (dyshidrotic eczema). This may be the first case of this presentation to be reported.

Case presentation A 50-year-old Caucasian female with a Background of $3 \mathrm{~mm}$ invasive ductal carcinoma of left breast presented with extensive vesicles and blistering on palms of hands and feet following treatment with trastuzumab [figure 1. She had wide local excision of a $3 \mathrm{~mm}$ invasive ductal carcinoma of left breast in January 2018 and sentinel lymph node biopsy which revealed a grade 2 , HER 2 positive, node negative, breast cancer. She received 6 cycles of adjuvant chemotherapy with epirubicin and cyclophosphamide followed by 15 fractions of 40 Gy adjuvant radiotherapy. Thereafter she was commenced on targeted therapy with trastuzumab. After the 2nd cycle of trastuzumab she developed multiple small vesicular eruptions predominantly on the hands and feet, which worsened by the 3 rd cycle. These were diagnosed by a dermatologist as extensive pompholyx, which was treated with topical steroid, emollient, potassium permanganate and fludroxycortide tape on the palms. Her swab cultures grew Staphylococcus aureus and betahemolytic streptococcus group-A, which was subsequently treated with intravenous flucloxacillin with a modest response.

Discussion Trastuzumab is a humanized monoclonal antibody that blocks HER2 receptors, undermining HER2 signaling in HER2 over-expressing breast cancers. The pathogenesis of pompholyx ${ }^{2}$ is not well understood thus it is difficult to establish a direct connection with trastuzumab at this stage. According to some immunohisto chemistry studies, strong presence of CD3, CD8, CD45 as well as anti-human antibodies are found in pompholyx whereas an immune mediated T-Cell lymphocyte-driven response in suppressing tumor growth in cancer has extensively been described. We plan to

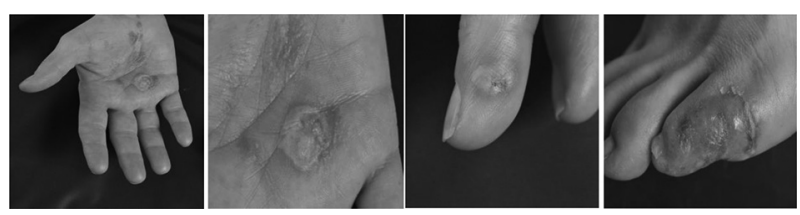

Abstract 2 Figure 1 Multiple cutaneous lesions on the palm, finger and toe. Images consented for teaching in the healthcare context. () University Hospitals of Leicester NHS Trust (Dept of Medical Illustration) 